\title{
Spectroscopic Study of the Optical Counterpart to the Fast X-ray Transient IGR J17544-2619 Based on Observations at the 1.5-m RTT-150 Telescope
}

\author{
I. F. Bikmaev ${ }^{1,2^{*}}$, E. A. Nikolaeva ${ }^{1,2^{*}}$, V. V. Shimansky ${ }^{1}$, A. I. Galeev ${ }^{1,2}$, \\ R. Ya. Zhuchkov ${ }^{1,2}$, E.N. Irtuganov ${ }^{1,2}$, S. S. Melnikov ${ }^{1,2}$, N. A. Sakhibullin ${ }^{1,2}$, \\ S.A.Grebenev ${ }^{3}$, and L.M. Sharipova ${ }^{4}$ \\ ${ }^{1}$ Kazan (Volga Region) Federal University, Kazan, Russia \\ ${ }^{2}$ Academy of Sciences of Tatarstan, Kazan, Russia \\ ${ }^{3}$ Space Research Institute, Russian Academy of Sciences, Moscow, Russia \\ ${ }^{4}$ Crimean Astrophysical Observatory, Russian Academy of Sciences, Nauchnyi, Crimea, Russia \\ Received September 26, 2016
}

\begin{abstract}
We present the results of our long-term photometric and spectroscopic observations at the Russian-Turkish RTT-150 telescope for the optical counterpart to one of the best-known sources, representatives of the class of fast X-ray transients, IGR J17544-2619. Based on our optical data, we have determined for the first time the orbital and physical parameters of the binary system by the methods of Doppler spectroscopy. We have computed theoretical spectra of the optical counterpart by applying non-LTE corrections for selected lines and obtained the parameters of the stellar atmosphere and the optical star $\left(T_{\text {eff }}=33000 \mathrm{~K}, \log g=3.85, R=9.5 R_{\odot}\right.$, and $\left.M=23 M_{\odot}\right)$. The latter suggest that the optical star is not a supergiant as has been thought previously.
\end{abstract}

DOI: $10.1134 / \mathrm{S} 1063773717100012$

Key words: high-mass X-ray binaries, fast X-ray transients, IGR J17544-2619.

\section{INTRODUCTION}

The source IGR J17544-2619 belongs to supergiant fast X-ray transients (SFXTs), a new population of Xray objects discovered by the INTEGRAL observatory (Sunyaev et al. 2003b; in't Zand 2005; Negueruela et al. 2006; Sguera et al. 2006; Grebenev 2009; Romano et al. 2014). X-ray binaries containing an early-type (OB) supergiant and a neutron star with a strong magnetic field (an X-ray pulsar) are representatives of this population.

Supergiants are characterized by a strong outflow of material (with a rate $\sim 10^{-6}-10^{-5} M_{\odot} \mathrm{yr}^{-1}$ ). Such a dense stellar wind must supply sufficient material to maintain spherically symmetric accretion onto the neutron star. Therefore, one would think that these binary systems must be powerful X-ray sources with a luminosity $\sim 10^{37} \mathrm{erg} \mathrm{s}^{-1}$ (Grebenev and Sunyaev 2007). Actually, this is not the case. Most of the time SFXTs have a very low luminosity $10^{32}-10^{33} \mathrm{erg} \mathrm{s}^{-1}$ and are not accessible for detection

*e-mail: ibikmaev@yandex.ru, evgeny.nikolaeva@gmail.com by wide-field X-ray telescopes. However, they occasionally flare up for a short ( $\lesssim 1$ day) time, becoming the brightest objects in the X-ray sky. Their luminosity rises by $4-6$ orders of magnitude in a few minutes. Various models have been proposed to explain the so unusual properties of SFXTs (the absence of persistent emission, the brevity of outbursts, the huge range of luminosity variations). Historically the first model of a clumpy stellar wind from a supergiant (in't Zand et al. 2004) did not explain the observed wide range of luminosity variations, the model of a highly asymmetric stellar wind (like the wind from Be stars, Sidoli et al. 2007) did not explain the outburst brevity, and the model of a magnetic barrier (Bozzo et al. 2008) suggested an extremely strong neutron star magnetic field. The model by Grebenev and Sunyaev (2007) (see also Bozzo et al. 2008), where the accretion of matter is halted by a centrifugal barrier at the magnetospheric boundary of a rapidly spinning neutron star (the propeller effect), while the outbursts are explained by the temporary overcoming of this barrier due to lo- 


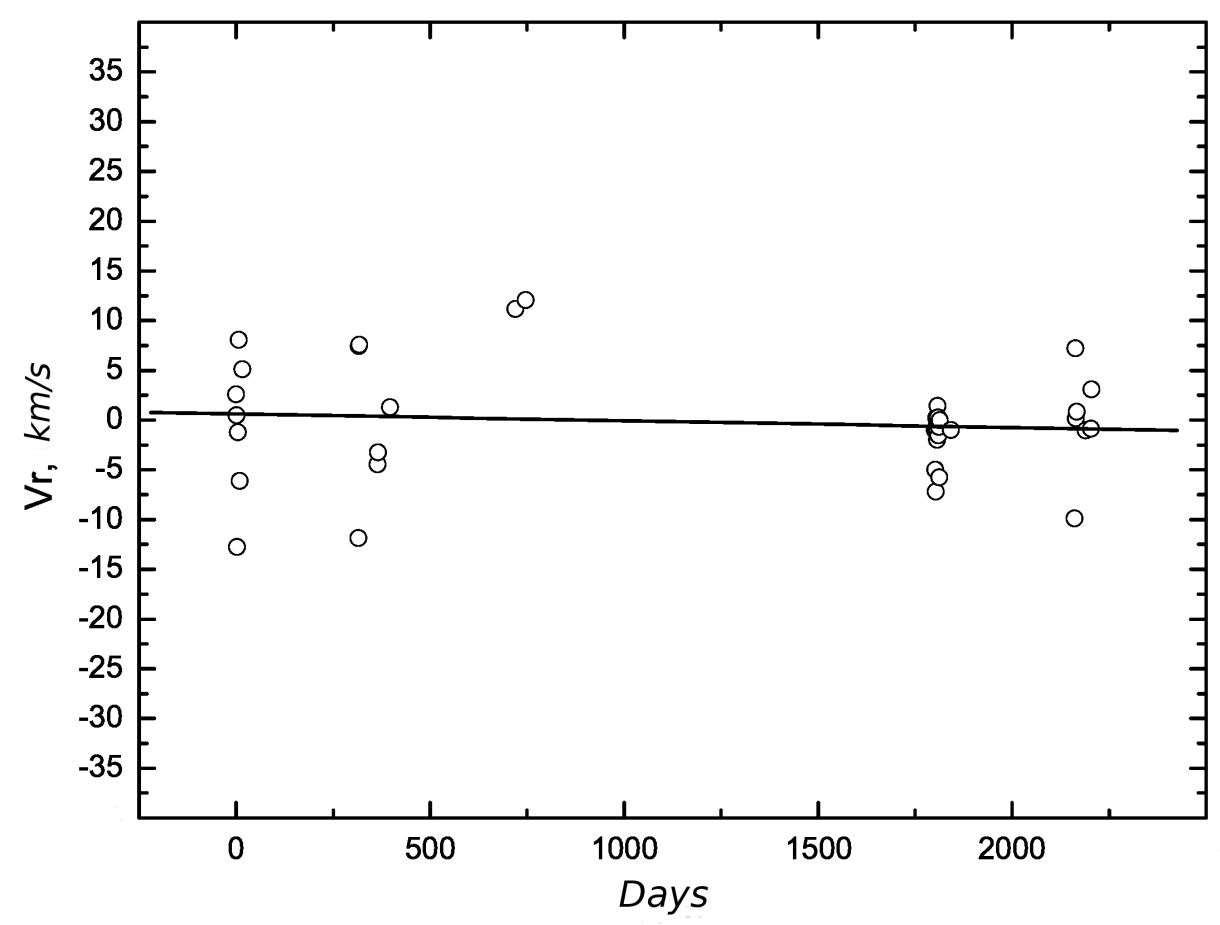

Fig. 1. Scatter of radial velocities from the interstellar NaD lines.

cal fluctuations of the stellar wind density and velocity, and the model by Shakura et al. (2014), which suggests quasi-spherical subsonic (settling) accretion onto a slowly spinning neutron star in a steady state and an enhancement of accretion during outbursts due to the development of Rayleigh-Taylor instability at the magnetospheric boundary, remain topical and are widely discussed. However, the latter model requires that the stellar wind be strongly magnetized and the neutron star spin be too slow $\left(P_{s} \gtrsim 1000 \mathrm{~s}\right)$.

The source IGR J17544-2619 was discovered during an X-ray outburst on September 17, 2003, (Sunyaev et al. 2003b) with the IBIS/ISGRI gamma-ray telescope (Ubertini et al. 2003; Lebrun et al. 2003) onboard the INTEGRAL observatory (Winkler et al. 2003). Recurrent outbursts (Grebenev et al. 2003, 2004) showed that the source is similar to the other known transient XTE J1739-302 (Sunyaev et al. 2003a; Smith et al. 2003, 2006), from which such outbursts were observed, and allowed one to talk about the discovery of a new population of X-ray sources, SFXTs. The source IGR J17544-2619 became a canonical representative of this population; it was used to verify the properties of SFXTs, to investigate their behavior, and to construct their models. The properties of this transient are indeed unique. For example, an outburst of IGR J17544-2619 with a record luminosity $\sim 3 \times 10^{38}$ $\operatorname{erg~s}^{-1}$ was detected on October 10, 2014, thereby ex- tending the range of its luminosity variations to six orders of magnitude (Romano et al. 2015). Based on NuSTAR satellite observations of the source, Bhalerao et al. (2015) have measured for the first time the magnetic field strength of the neutron star in SFXTs, $\sim 1.5 \times 10^{12} \mathrm{G}$ (from a cyclotron line $\left.h \nu_{c} \simeq 17 \mathrm{keV}\right)$; this value is typical for the neutron stars in X-ray binaries. The optical counterpart to IGR J17544-2619 was classified as an O9Ib supergiant with a mass of 25-28 $M_{\odot}$ (Pellizza et al. 2006) and a radius in the range 12.7 $R_{\odot}<R<26.6 R_{\odot}$ (Rahoui et al. 2008). Based on the minimum radius, using the previously estimated masses of the supergiant, 25-28 $M_{\odot}$, and the compact object, $1.4 M_{\odot}$, and taking into account the absence of regular outbursts, suggesting that there is no Roche lobe overflow in the binary system, Clark et al. (2009) imposed a constraint on the orbital eccentricity, $e \lesssim 0.4$. Based on archival INTEGRAL data and using the Lomb-Scargle method (Lomb 1976; Scargle et al. 1982), the authors also determined the orbital period of the binary, $P_{b} \simeq(4.926 \pm 0.001)$ days. Subsequently, the period has been repeatedly confirmed (for various ephemerides) on the basis of X-ray data: $P_{b}=(4.9278 \pm 0.0002)$, MJD 53732.632 (Drave et al. 2012); $P_{b}=(4.9272 \pm 0.0004)$ days, MJD 55924.271 (Drave et al. 2014); and $P_{b}=(4.92693 \pm 0.00036)$ days, MJD 53732.65 (Smith 2014). However, as yet nobody has determined it directly from optical data. In this 


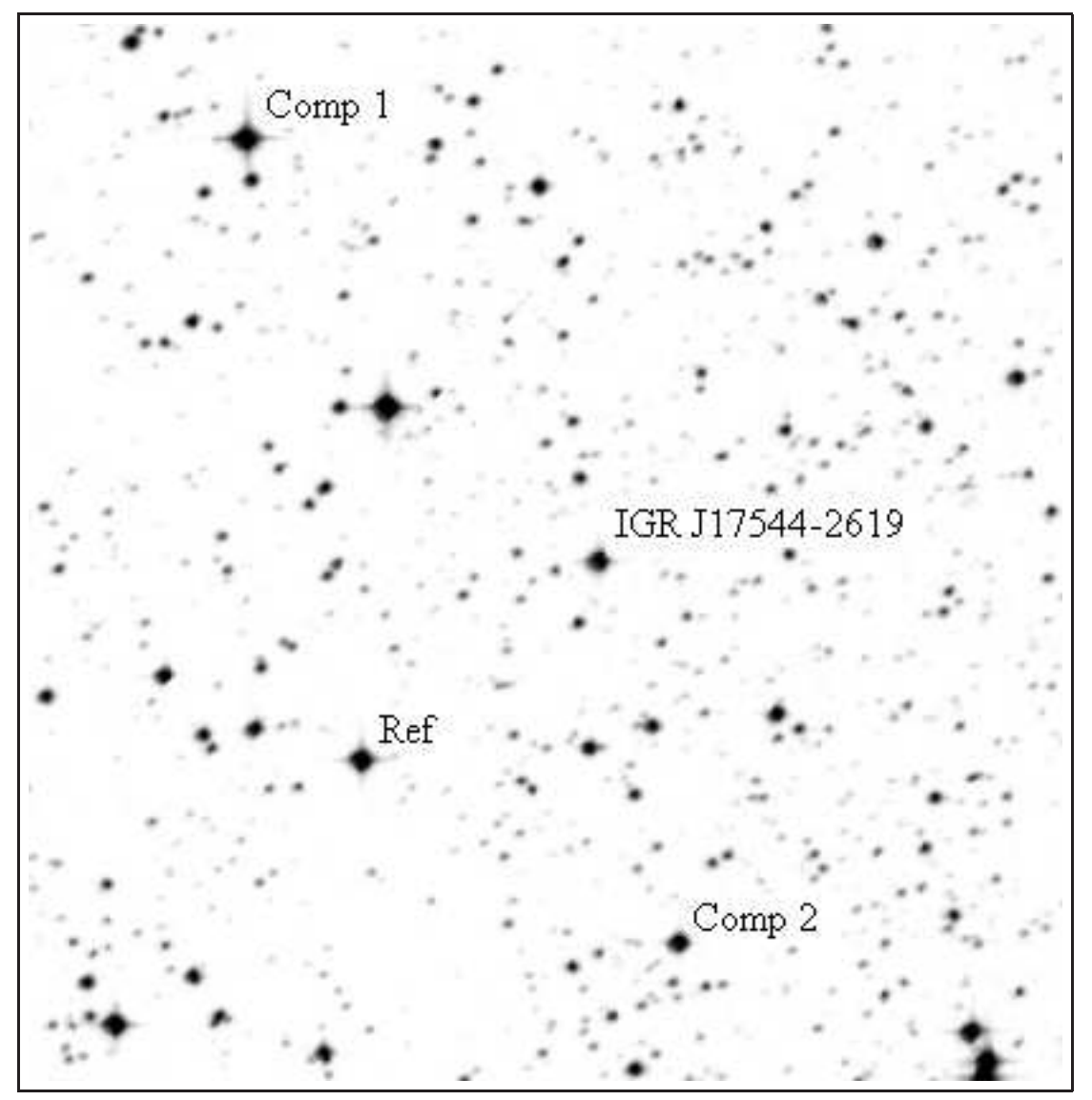

Fig. 2. Comparison stars Comp 1 (TYC 6849-1825-1) and Comp $2\left(\alpha=17^{\mathrm{h}} 54^{\mathrm{m}} 22^{\mathrm{s}} .578, \delta=-26^{\circ} 22^{\prime} 49^{\prime \prime} .43\right)$, reference star Ref $\left(\alpha=17^{\mathrm{h}} 54^{\mathrm{m}} 33^{\mathrm{s}} .457, \delta=-26^{\circ} 21^{\prime} 23^{\prime \prime} .74\right)$ and IGR J17544-2619.

paper we present the results of our long-term observations of the optical counterpart to this X-ray system at the Russian-Turkish RTT-150 telescope. Based on their analysis, we solved this problem.

Table 1. $B, V, R$, and $I$ magnitudes of the standard and reference stars

\begin{tabular}{ccccc}
\hline Star & $B$ & $V$ & $R$ & $I$ \\
\hline SA 104_598 & 12.585 & 11.479 & 10.809 & 10.263 \\
Ref & 13.08 & 12.18 & 11.71 & 11.25 \\
\hline
\end{tabular}

\section{OBSERVATIONS AND DATA ANALYSIS}

The photometric observations of the object were begun with the 1.5-m Russian-Turkish RTT-150 telescope at the TUBITAK National Observatory (Turkey) in 2003 shortly after the discovery of the source. Unfortunately, although dozens of X-ray outbursts have already been recorded from the source (see, e.g., Romano et al. 2013), none of them was detected during the observations being discussed. From 2003 to 2006 an ANDOR $(2048 \times 2048$ pixels $)$ CCD array was used for our photometry. Since 2007, after the identification of the object with an optical star (Pellizza et al. 2006), the photometric and spectroscopic observations have been performed with the TFOSC instrument using a medium-resolution $(2.5 \AA)$ echelle mode. The duration of each exposure was $30 \mathrm{~min}$. The spectra taken during the night were summed to increase the signal-to-noise ratio $(\mathrm{S} / \mathrm{N})$ in our data. The mean $\mathrm{S} / \mathrm{N}$ ratio of the summed spectrum was $70-120$. The spectra span the wavelength range $\lambda 4400-9150 \AA$. The photometry was carried out in the $B, V, R, I$, and white filters.

The reduction of the spectra was performed in the DECH software package (Galazutdinov 1992, 2007) and consisted of the following standard operations: bias subtraction, spectrum extraction, cosmic-ray particle and defective pixel removal, continuum placement, wavelength calibration, and spectral line radial velocity measurements. The radial velocities were measured by the cross-correlation method from the HeI $\lambda 5876,6678$, and $7065 \AA$ lines, because the hydrogen line profiles are severely distorted by the stellar wind from an $\mathrm{O}$ star. We controlled the instrumental accuracy of our measurements based on the radial ve- 
Table 2. $B, V, R$, and $I$ magnitudes of IGR J17544-2619

\begin{tabular}{lcccc}
\hline Data source & $B$ & $V$ & $R$ & $I$ \\
\hline \hline This paper (July 18, 2015) & $14.51 \pm 0.02$ & $12.77 \pm 0.01$ & $11.61 \pm 0.01$ & $10.35 \pm 0.01$ \\
This paper (June 30, 2016) & $14.52 \pm 0.01$ & $12.79 \pm 0.01$ & $11.63 \pm 0.01$ & $10.38 \pm 0.01$ \\
Pellizza et al. (2006) & $14.44 \pm 0.05$ & $12.65 \pm 0.05$ & $<11.9$ & - \\
Zacharias et al. (2012) & $14.71 \pm 0.01$ & $12.94 \pm 0.01$ & $12.10 \pm 0.07$ & - \\
DENIS databasea & - & - & - & $10.38 \pm 0.01$ \\
\hline a The DENIS database, the 3rd release, the CDS/ADC Collection of Electronic \\
Catalogues, 2263, 0 (2005).
\end{tabular}

Table 3. Radial velocities $\left(\mathrm{km} \mathrm{s}^{-1}\right)$

\begin{tabular}{|c|c|c|c|c|c|c|c|c|c|}
\hline Date & HJD & Phase $\phi$ & $\overline{V_{r}}$ & $\delta V_{r}$ & Date & HJD & Phase $\phi$ & $\overline{V_{r}}$ & $\delta V_{r}$ \\
\hline Aug. 1, 2007 & 2454314. & 0.95910 & 22.8 & 2.7 & July 18,2012 & 2456127.3 & 0.90505 & 25.8 & 6.5 \\
\hline Aug. 2, 2007 & 2454315.3842 & 16297 & -2.6 & 6.0 & Aug. 16, 2012 & 2456156.2997 & 557 & 32.0 & 3.6 \\
\hline Aug. 3, 2007 & 2454316.3704 & 363 & -23.5 & 3.7 & July 1, 2013 & 2456475.4444 & 0.55750 & 22.4 & 8.1 \\
\hline Aug. 5, 2007 & 2454318 . & 25 & 29.3 & 3.4 & July 3, 2013 & 2456477.4579 & 16 & 24.2 & .0 \\
\hline Aug. 8, 2007 & 2454321 . & 0.36649 & 7.5 & 5.7 & July 4, 2013 & 2456478.4011 & 760 & -20.1 & 3.8 \\
\hline Aug. 17, 2007 & 2454330.3103 & 19231 & 0.3 & 1.8 & July 6,2013 & 2456480.4159 & & 17.5 & 4.9 \\
\hline June 11, 2008 & 2454629.3840 & 0.89073 & 37.4 & 2.7 & July 29, 2013 & 2456503.3269 & 640 & -17.1 & 5.2 \\
\hline June 12, 2008 & 2454630.5159 & 45 & -10.4 & 4.8 & Aug. 12, 2013 & 2456517 & 92 & 2.9 & 5.5 \\
\hline June 13, 2008 & 24546 & .30969 & 6.8 & 3.7 & Aug. 14, 2013 & 245651 & 367 & 6.6 & 1.8 \\
\hline July 30,2008 & 2454 & 82040 & 33.4 & 4.7 & June 23, 2014 & 2456832.3755 & 339 & 7.8 & 4.0 \\
\hline July 31 , & 245 & & 2.9 & 2.6 & 14 & & & -5.5 & .9 \\
\hline Aug. 31, 2008 & 2454710.2850 & .30997 & -8.0 & 4.8 & 26,2014 & 2456835.4433 & 102 & 21.6 & 3.3 \\
\hline July 21 , & 2 & & -7.1 & 4.9 & $\mathrm{~J}$ & & & 34.1 & 6 \\
\hline Aug. 16, 2009 & 2455060.3641 & .36020 & -2.4 & 4.2 & June 28, 2014 & 2456837.4328 & 0.02479 & -0.9 & 3.5 \\
\hline July 6 , & 24561 & & 9.2 & 3.6 & 1,2014 & 2456840 & & 7.0 & 1.8 \\
\hline July 7, 2012 & 2456116.4460 & 706 & 32.2 & 5.5 & ly 2,2014 & 2456841.4278 & 560 & 24.0 & 1.4 \\
\hline July 8, 2012 & 245611 & $0 . \zeta^{2}-x$ & 42.3 & 5.3 & 24,2014 & 2456863.3582 & 48 & -19.2 & 5.0 \\
\hline July 9, r & 245611 & 9 & -3.0 & 4.5 & July 26,2014 & 2456865.3656 & 387 & 23.8 & 0.6 \\
\hline July 10, 2012 & 2456119.3389 & 0.28420 & 4.8 & 3.4 & July 28,2014 & 2456867.3430 & 521 & -26.2 & 2.7 \\
\hline July 11, 2012 & 2456120.3559 & 0.4 & 24.5 & 5.2 & 21,2015 & 2457225.3542 & 28 & 26.1 & 5.6 \\
\hline July 12, 2012 & 2456121.3764 & 0.69772 & 38.7 & 7.1 & June 30, 2016 & 2457570.4555 & 0.79525 & 24.8 & 0.9 \\
\hline July 13, 2012 & 2456122.3747 & 0.90 & 28.5 & 1.7 & July 1, 2016 & 2457571.4399 & 0.99503 & -4.5 & 7.6 \\
\hline July 14, 2012 & 2456123.3463 & 0.09753 & -5.7 & 5.0 & July 2, 2016 & 2457572.4317 & 0.19633 & -13.7 & 4.4 \\
\hline July 15, 2012 & 2456124.4007 & 0.31 & -6.9 & 6.0 & July 3,2016 & 2457573.4164 & 0.3 & -13.6 & 2.6 \\
\hline July 16, 2012 & 2456125.3193 & 0.49794 & 31.1 & 6.7 & July 6,2016 & 2457576.3952 & 0.00073 & -14.5 & 7.2 \\
\hline
\end{tabular}

Table 4. Orbital parameters of IGR J17544-2619

\begin{tabular}{c|c}
\hline Parameters & Values \\
\hline$P_{b}^{\mathrm{b}}$ & $4.927206 \pm 0.0002$ \\
$T^{\mathrm{c}}$ & $54314.6 \pm 0.1$ \\
$e^{\mathrm{d}}$ & $0.42 \pm 0.07$ \\
$\omega^{\mathrm{e}}$ & $271 \pm 12$ \\
$K^{\mathrm{f}}$ & $21 \pm 2$ \\
$\gamma^{\mathrm{g}}$ & $9 \pm 1$ \\
\hline
\end{tabular}

\footnotetext{
a Found using A.A. Tokovinin's ORBIT code.

b The orbital period, days.

c The time of periastron passage (JD-2 400000$)$, days.

d The orbital eccentricity.

e The longitude of periastron in degrees.

$\mathrm{f}$ The semi-amplitude of the radial velocity curve, $\mathrm{km} \mathrm{s}^{-1}$.

$\mathrm{g}$ The center-of-mass velocity, $\mathrm{km} \mathrm{s}^{-1}$.
} 


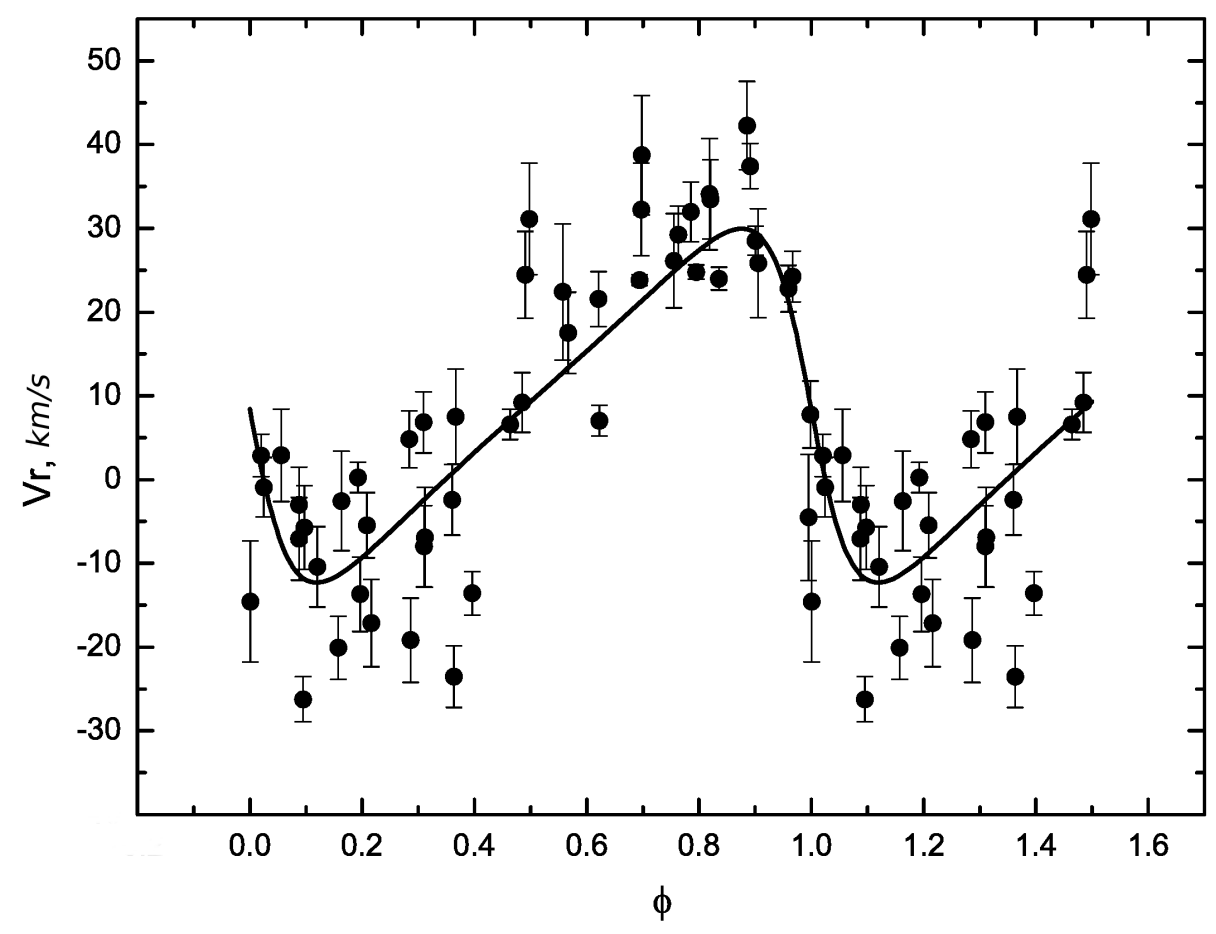

Fig. 3. Radial velocity curve of the optical counterpart to IGR J17544-2619.

locity measurements of the interstellar sodium lines (Na I $\lambda 5896,5890 \AA$ ). The rms error in the radial velocities of the $\mathrm{Na}$ lines was $6 \mathrm{~km} \mathrm{~s}^{-1}$ (Fig.1) and was subsequently used as the instrumental uncertainty.

\section{RESULTS}

Photometry

The magnitude of the optical star being investigated was measured relative to the reference star Ref (Fig.22); whose magnitude was measured using the Landolt photometric standard SA 104_598 (Table 1). No photometric variability of the object was detected at an accuracy level of $\pm 0{ }^{\mathrm{m}} 02$ controlled with using the comparison stars Comp1 and Comp2. This is evidenced by the long series of their observations performed in the white filter. The measured $B, V, R$, and $I$ magnitudes on different dates of observations (July 18, 2015, and June 30, 2016) also lie within the error limits and are in good agreement with the previously published data (Table 2).

\section{Determining Orbital Parameters of the Binary System}

Based on the spectra taken in 2007-2011, we measured the radial velocities of the optical counterpart to IGR J17544-2619 (Table 3) and then made an attempt to find the orbital period of the binary system. The derived preliminary period, 12.18 days (Nikolaeva et al. 2013), contradicted the period found by Clark et al. (2009) from X-ray data, $P_{b} \simeq(4.926 \pm 0.001)$ days. Therefore, the observations were continued, and in 2012 we obtained a continuous 13-day series of spectra whose analysis gave a period consistent with Clark's estimate. Followup observations allowed the period to be determined with an even higher accuracy. The orbital period was determined by the LaflerKinman (1965) and Deeming (1975) methods using V.P. Goransky's WinEfk code from the spectroscopic data spanning the period of observations 2007-2016. The tables of possible periods can be found in Nikolaeva et al. (2017). The orbital parameters of the binary system were determined from the best period found using A.A. Tokovinin's ORBIT code by the gradient-descent method (Table 4). The derived radial velocity curve is presented in Fig. 3. Given the period and the semi-amplitude of the radial velocity curve, we calculated the mass function of the binary system $f(m)=(3.6 \pm 1.0) \times 10^{-3} M_{\odot}$ and the line-ofsight projection of the semimajor axis of the optical counterpart $a_{v} \sin i=(0.00871 \pm 0.00086)$ AU.

\section{Determining the Mass of the Optical Counterpart}

The first steps in determining the mass of the optical star of IGR J17544-2619 were taken in Pellizza et al. (2006), where the star was found from the ratio of the He II $\lambda 4541 \AA / \mathrm{HeI} \lambda 4471 \AA$ line intensities to be of spectral type O9, while its luminosity class Ib was determined based on the presence of the He II $\lambda 4686 \AA$ line, which is a criterion for this luminosity class (the 

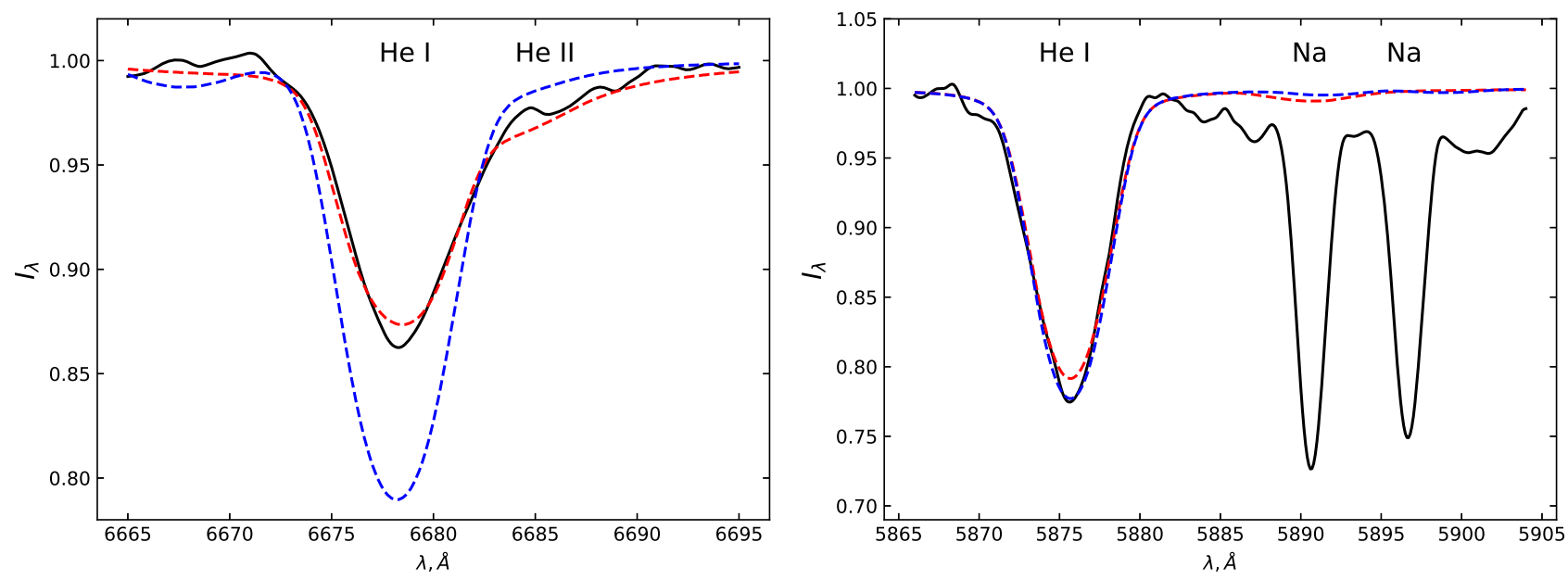

Fig. 4. Observed and theoretical HeI $\lambda 6678 \AA$ and HeII $\lambda 6683 \AA$ line profiles. The observed spectrum is indicated by the black solid line; the red dashed line is the theoretical spectrum that is in best agreement with the observed one, $T_{\text {eff }}=33000 \mathrm{~K}$ and $\log g=3.85$; the blue dashed line is the synthetic spectrum typical for a supergiant with $T_{\text {eff }}=29000$ $\mathrm{K}, \log g=3.20$. The distribution of colors on the succeeding graphs is analogous.

Fig. 5. Observed and theoretical profiles of the HeI $\lambda 5875 \AA$ line and the interstellar NaD lines demonstrating the spectral resolution.

spectral atlas of Walborn and Fitzpatrick 1990). Thus, the mass of the optical counterpart was limited by the range $25-28 M_{\odot}$.

Gimenez-Garcia et al. (2016) estimated the mass of the supergiant, $(25.9 \pm 1.0) M_{\odot}$, by computing its synthetic spectra using the Potsdam Wolf-Rayet model atmosphere code. The effective temperature was calculated from the ratios of the He I / He II and Si III / Si IV lines, while $\log g_{\text {eff }}$ was derived from the broadened wings of the Balmer lines and He II lines. The stellar radius $R=17 R_{\odot}$ was determined from the luminosity and the effective temperature found from modeling; the mass of the optical counterpart was then calculated using $\log g_{\text {eff }}$.

In our paper we independently determined the physical parameters of the optical counterpart by studying the profiles of spectral lines forming in its atmosphere. For our analysis we selected the observed spectrum containing a maximum set of absorption lines in a wide wavelength range, $\lambda 5400-7080 \AA$, that had no appreciably contribution from the emission components, i.e., were undistorted by the wind outflows from the star. We computed theoretical normalized spectra for the optical counterpart to IGR J17544-2619 using the STAR code (Menzhevitski et al. 2014), which takes into account the blanketing in atomic and molecular lines and the non-LTE effects for selected atoms and ions (in our case, HI, HeI, HeII, CII, C III, and MgII). Hydrostatic, plane-parallel model stellar atmospheres with specified sets of parameters $T_{\text {eff }}, \log g,[\mathrm{He} / \mathrm{H}]$, and $[\mathrm{M} / \mathrm{H}]$ were computed using the ATLAS12 software (Castelli and Kurucz 2004) adapted to personal computers and kindly provided to us by V. Tsymbal. In the initial model computations we specified a solar chemical composition according to the data from Anders and Grevesse (1989), while in the final ones it was changed based on the abundance determinations for a number of elements when analyzing the observed spectrum (see below).

Based on the constructed models, when solving the transfer equation by Hermitte's method, we calculated the specific intensities of the optical emission emerging at three fixed angles $\theta^{\prime}$ relative to the normal to the stellar surface. We took into account all of the continuous absorption sources tabulated in STARDISK (Suleymanov 1992) and SPECTR (Shimansky et al. 2012) and $\sim 2000000$ lines from the lists by Kurucz (1994) and Castelli and Kurucz (2004). In our computations of the H I and He II line profiles we used the Vidal-Cooper-Smith (1973) and Griem (1960) theories. The profiles of the remaining lines were modeled by taking into account the thermal motion of atoms and the microturbulence with velocity $\xi_{\text {turb }}$, the natural damping, the Stark broadening according to the data from Kurucz and Furenlid (1979) and the van der Waals broadening according to Gray's formula (Unsold 1955). The departures from LTE in the stellar atmospheres were found by applying 22-level H I, 38-level 


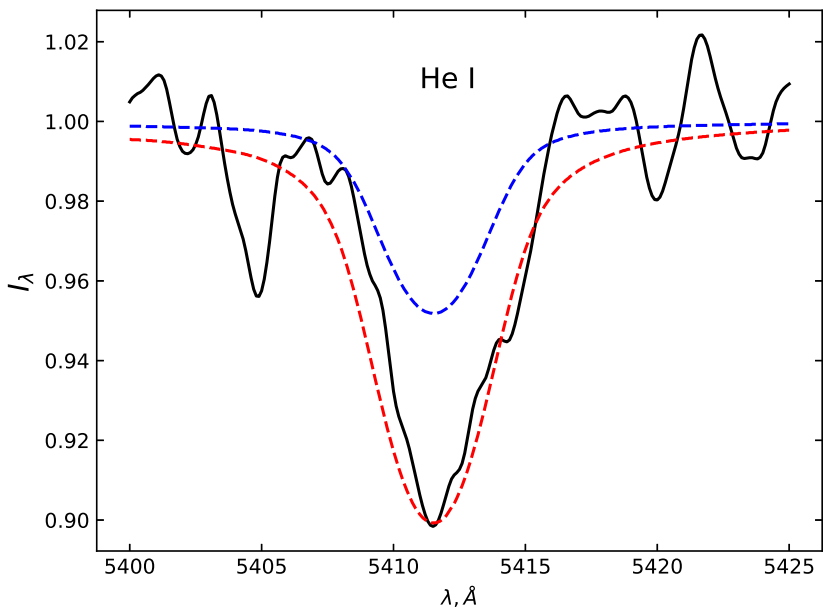

Fig. 6. Observed and theoretical He II $\lambda 5411 \AA$ line profiles.

HeI, 23-level HeII, 50-level CII, 94-level C III, 45level Mg II models and the NONLTE software package (Sakhibullin 1983) in the form of Menzel coefficients for the energy levels of a selected atom and were passed to the STAR code according to the technique developed by Shimansky (2012). The basic solar chemical composition was specified in accordance with the data from Anders and Grevesse (1989). Then, the stellar surface was divided into fields with a $2^{\circ}$ step in two orientation angles. For each field we found the area, the observer's visibility angle $\theta$, and the line-of-sight component of the rotational velocity. The stellar spectrum was computed by summing the intensities of the fields interpolated to the angle $\theta$ and shifted in wavelength in accordance with their radial velocity. The final spectrum was convolved with the response function of the spectrograph specified by a Gaussian with a FWHM corresponding to the spectral resolution.

A proper analysis of the observed spectrum with the determination of the binary parameters is possible under the condition of its normalization based on its comparison with the theoretical spectrum. Therefore, the renormalization was performed so as to accurately match the mean residual intensities in the observed and theoretical spectra in the segments that are definitely free from lines. The positions of these segments were chosen by analyzing the theoretical spectrum provided that the intensity exceeded $I_{\lambda}=0.98$ and that there were no unknown lines in the observed spectrum at a given wavelength. The observed spectrum contains a complex emission-absorption $H_{\alpha}$ profile, the HeI $\lambda 5875,6678,7065 \AA$, He II $\lambda 5411,6406,6526$, 6683, $6890 \AA$, C III $\lambda 5826 \AA$, C IV $\lambda 5801,5812 \AA$, O III $\lambda 5508,5592 \AA$ absorption lines, and the C III

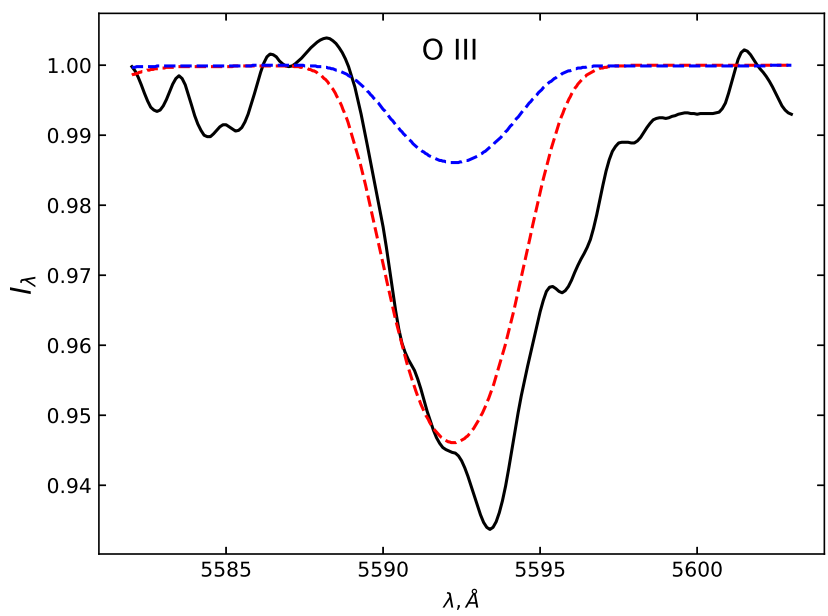

Fig. 7. Observed and theoretical O III $\lambda 5592 \AA$ line profiles.

$\lambda$ 5694, $5696 \AA$ emission blend. Obviously, the $H_{\alpha}$ profile is formed almost completely in the stellar wind and is unsuitable for analyzing the stellar characteristics. The remaining lines are formed in the stellar atmosphere and can be used to determine its parameters. The presence of helium and carbon lines in two successive ionization stages in the spectra theoretically allows us to investigate their ionization equilibria and to find the abundances of elements in the atmosphere of the star, its effective temperature $T_{\text {eff }}$, and surface gravity $\log g$ in a joint analysis. Note that the neglect of departures from LTE for C IV in our technique and the weakness of the C III and C IV lines can cause significant errors in constructing the C III/C IV equilibrium. Therefore, when analyzing the spectrum and determining the parameters of IGR J17544-2619, we focused our attention on a proper description of the observed HeI and He II line profiles, while the C III and C IV lines were used to find the carbon abundance and to check the results. However, it should be noted that the presence of C IV and O III lines in the stellar spectra unambiguously points to its effective temperature appreciably exceeding $T_{\text {eff }}=30000 \mathrm{~K}$.

The parameters and chemical composition of IGR J17544-2619 were obtained by the method of successive approximations until an optimal match between the observed and theoretical line profiles was achieved. The effective temperature was determined by the requirement that the unblended HeI $\lambda 5875$, $6678 \AA$ lines and the strongest unblended He II $\lambda 5411$ $\AA$ line be described simultaneously. The surface gravity was estimated by analyzing the profiles of the HeI $\lambda 5875,6678 \AA$ lines with different sensitivities to the adopted $\log g$. To find the microturbulent ve- 


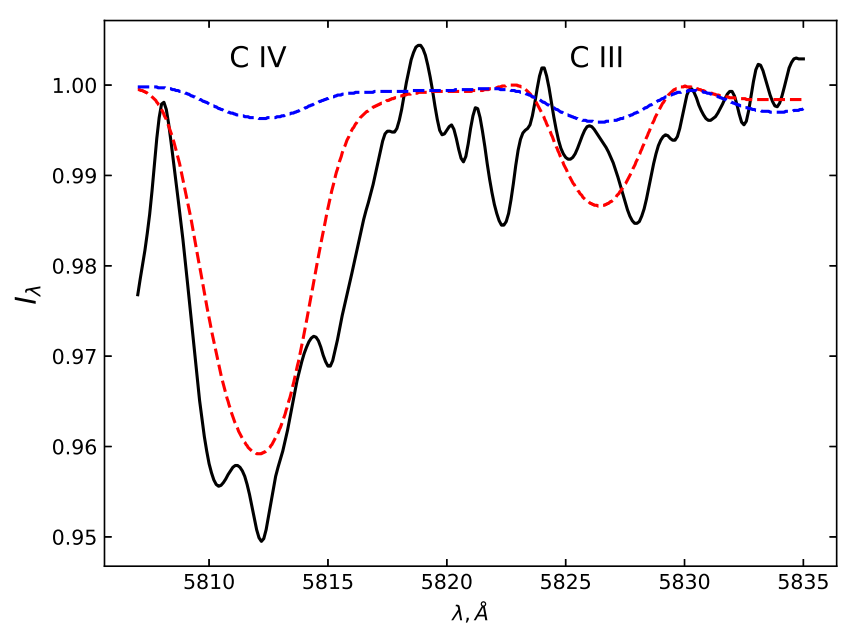

Fig. 8. Observed and theoretical C IV $\lambda 5812 \AA$ and C III $\lambda 5826 \AA$ line profiles.

locity $\xi_{t}$, we used the O III $\lambda 5508,5592 \AA$ and C IV $\lambda 5801,5812 \AA$ lines of different intensities. This approach allowed, on the whole, satisfactory agreement between the observed and theoretical spectra of the object to be achieved at the follo wing parameters: $T_{\text {eff }}=(33000 \pm 1000) \mathrm{K}, \log g=(3.85 \pm 0.15),[\mathrm{He} / \mathrm{H}]$ $=0.45 \operatorname{dex},[\mathrm{C} / \mathrm{H}]=0.4 \operatorname{dex},[\mathrm{N} / \mathrm{H}]=0.5 \operatorname{dex},[\mathrm{O} / \mathrm{H}]$ $=0.8 \mathrm{dex}, \xi_{t}=17 \mathrm{~km} \mathrm{~s}^{-1}$.

Figures 48 present the deepest HeI $\lambda 6678 \AA$ and He II $\lambda 6683 \AA$, HeI $\lambda 5875 \AA$, He II $\lambda 5411 \AA$, O III $\lambda 5592 \AA$, CIV $\lambda 5812 \AA$, and C III $\lambda 5826 \AA$ lines. The computed theoretical spectrum is presented in comparison with the observed spectrum and the theoretical one for atmospheric parameters typical for a supergiant, $T_{\text {eff }}=29000 \mathrm{~K}, \log g=3.20 \mathrm{dex}$, and $\xi_{t}=25 \mathrm{~km} \mathrm{~s}^{-1}$, and determined in Gimenez-Garcia et al. (2016). Obviously, using the previously published parameters does not allow a number of features in the observed spectrum, in particular, the O III, C IV, He I $\lambda 6678 \AA$, He II $\lambda 5411 \AA$ lines and a number of weaker He II lines, to be described. Therefore, we believe the set of stellar atmosphere parameters we found to be more reliable and to admit a proper match of the observed and theoretical spectra. Note that the enhanced abundances of helium and light elements we found 1 can be associated with the complex evolution of IGR J17544-2619: these elements could be synthesized in the interiors of the primary component after

\footnotetext{
${ }^{1}$ Note that the accuracy of the helium abundance determination when analyzing the lines of the HeI and He II ionization stages with a complete allowance for the departures from LTE is about 0.15 dex. The $\mathrm{C}$ abundance derived from the lines of two ionization stages (one in non-LTE) has errors of about 0.22 dex; the $\mathrm{O}$ abundance has an error up to 0.25 dex. Therefore, on the whole, the $\mathrm{C}$ and $\mathrm{O}$ overabundances we found may be deemed real.
}

its transformation into a supergiant, i.e., when the binary system was passing the common-envelope phase. The partial transfer of matter to the secondary component (the now observed optical star) occurring at this time could contribute to its enrichment by newly synthesized elements. Note that similar overabundances of light elements have previously been found in other high-mass binaries with relativistic components (for example, in V1357 Cyg, see Shimansky et al. 2012).

Figure 9 shows the position of the optical counterpart to IGR J17544-2619 on the $\log T_{\text {eff }}-\log \left(L / L_{\odot}\right)$ diagram in comparison with the evolutionary tracks (Martins and Palacios 2012) for high-mass stars. We determined the stellar luminosity iteratively based on the previously estimated $T_{\text {eff }}=(33000 \pm 1000) \mathrm{K}$ and $\log g=(3.85 \pm 0.15)$. As a result, we found the full set of fundamental stellar parameters: the bolometric luminosity $\log \left(L / L_{\odot}\right)=4.98 \pm 0.10$, the mass $M=(23 \pm 2) M_{\odot}$, and the radius $R=(9.5 \pm 1.5) R_{\odot}$.

\section{DISCUSSION}

One of the most important achievements of the INTEGRAL gamma-ray observatory is the discovery of a hitherto unknown population of Galactic X-ray binaries called Supergiant Fast X-ray Transients. As has already been said, these are binaries consisting of a neutron star with a strong magnetic field (an $\mathrm{X}$-ray pulsar) and an early-type supergiant. Only a few X-ray binaries with supergiants were known before their discovery. These were all quasi-persistent $\mathrm{X}$-ray sources emitting through accretion from the supergiant stellar wind, as is expected for such binaries. It was unclear why they are so few. With the discovery of SFXTs the number of binaries with supergiants has increased noticeably and already reaches dozens. According to the SFXT model proposed by Grebenev and Sunyaev (2007), the reason why persistently emitting binary systems with supergiants are scarcely observed is the action of a centrifugal barrier at the magnetospheric boundary of the neutron star (the propeller effect, see Illarionov and Sunyaev 1975) halting the accretion, while the outbursts of SFXTs are associated with the temporary overcoming of this barrier due to a local increase in the density or a decrease in the velocity of the stellar wind. Although qualitatively the model explains well the observed phenomenon, a reliable determination of the orbital and other parameters of the binary system for a number of SFXTs, which is quite difficult to do, is needed to test it quantitatively.

In this paper we presented the results of our longterm study of the optical counterpart (companion star) to one of the canonical SFXTs, IGR J17544-2619, at RTT-150. The orbital and physical parameters of the binary system were determined reliably by the meth- 


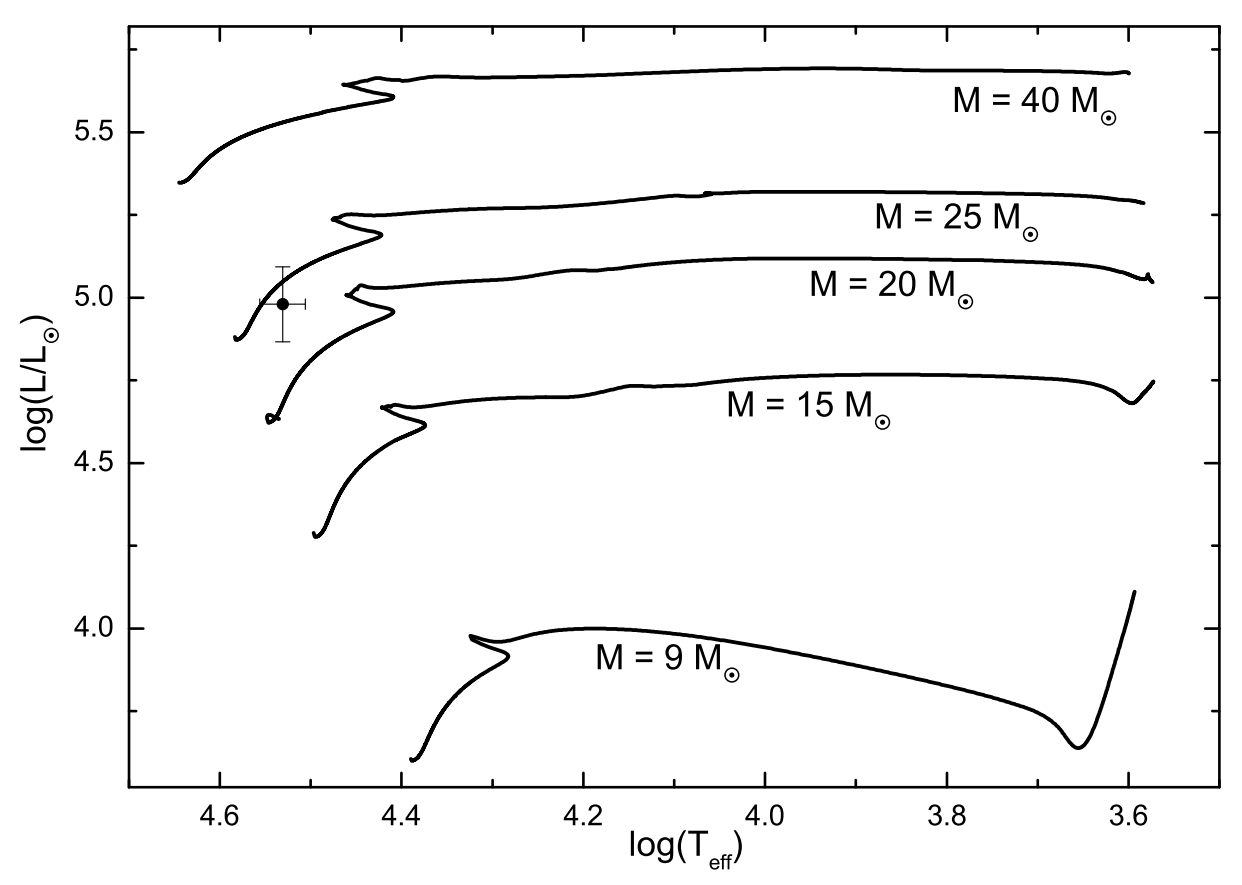

Fig. 9. Position of the optical counterpart to IGR J17544-2619 on the diagram $\log T_{\text {eff }}-\log \left(L / L_{\odot}\right)$ in comparison with the evolutionary tracks.

ods of Doppler spectroscopy and by modeling its synthetic spectra. The derived mass $M=(23 \pm 2) M_{\odot}$ and radius $R=(9.5 \pm 1.5) R_{\odot}$ of the optical star differ from the previously published values and correspond to an O9 IV-V star that "sits" more deeply in its Roche lobe. At the neutron star mass $M_{X}=1.4 M_{\odot}$, according to Kepler's law, the separation between the components is $a \simeq 35.5 R_{\odot}$, while the size of the Roche lobe of the optical counterpart, $R_{r}=a\left[0.38+0.2 \log \left(M / M_{X}\right)\right] \simeq$ $22 R_{\odot}$ (Paczynski 1971), is more than twice its actual radius $R=(9.5 \pm 1.5) R \odot$. 2 . Contrary to the prevalent opinion, the optical star of the binary does not belong to supergiants. Nevertheless, it is close to them in its many manifestations. In particular, the calculated effective temperature $T_{\text {eff }}=(33000 \pm 1000) \mathrm{K}$ is sufficient for the formation of an intense stellar wind $\dot{M}_{W} \simeq(1-4) \times 10^{-7} M_{\odot} \mathrm{yr}^{-1}$ (Vink et al. 2001; van Buren 1985) capable of providing an accretion rate onto the neutron star

$$
\begin{gathered}
\dot{M}=\frac{1}{4}\left(\frac{r_{a}}{a}\right)^{2} \dot{M}_{W} \simeq \\
\simeq 1.6 \times 10^{-10}\left(\frac{\dot{M}_{W}}{2 \times 10^{-7} M_{\odot} \mathrm{yr}^{-1}}\right) \times
\end{gathered}
$$

\footnotetext{
${ }^{2}$ Since the orbit is highly elliptical, the minimum distance between the components and the minimum radius of the Roche lobe are $\sim 20.6 R_{\odot}$ and $\sim 12.8 R_{\odot}$, thus, at periastron the Roche radius exceeds the radius of the star by only $\sim 30 \%$. The rate of accretion from the wind at periastron is $\sim 3$ times higher than the average one in the orbit (see below).
}

$$
\times\left(\frac{v_{W}}{500 \mathrm{~km} \mathrm{~s}^{-1}}\right)^{-4} M_{\odot} \mathrm{yr}^{-1},
$$

where $v_{W}$ is the presumed stellar wind velocity and $r_{a}=2 G M_{X} / v_{W}^{2}$ is the matter capture radius. If the gravitational energy of the accreting matter $G M_{X} \dot{M} / R_{X} \simeq 0.2 \dot{M} c^{2}$ is completely reprocessed into radiation, then the $\mathrm{X}$-ray luminosity of the binary can reach

$$
\begin{aligned}
L_{X}= & 1.8 \times 10^{36}\left(\frac{\dot{M}_{W}}{2 \times 10^{-7} M_{\odot} \mathrm{yr}^{-1}}\right) \times \\
& \times\left(\frac{v_{W}}{500 \mathrm{~km} \mathrm{~s}^{-1}}\right)^{-4} \mathrm{erg} \mathrm{s}^{-1} .
\end{aligned}
$$

According to Grebenev and Sunyaev (2007), such a situation takes place only when the spin period of the neutron star $P_{s}$ exceeds the so-called equilibrium period (Grebenev 2009)

$$
\begin{gathered}
P_{s}^{*} \simeq 0.98\left(\frac{P_{b}}{5 \text { days }}\right)^{37 / 21} \times \\
\times\left(\frac{B}{10^{12} \mathrm{G}}\right)^{8 / 7}\left(\frac{v_{W}}{500 \mathrm{~km} \mathrm{~s}^{-1}}\right)^{44 / 7} \times \\
\times\left(\frac{\dot{M}_{W}}{1 \times 10^{-5} M_{\odot} \mathrm{yr}^{-1}}\right)^{-4 / 7} \mathrm{~s} .
\end{gathered}
$$




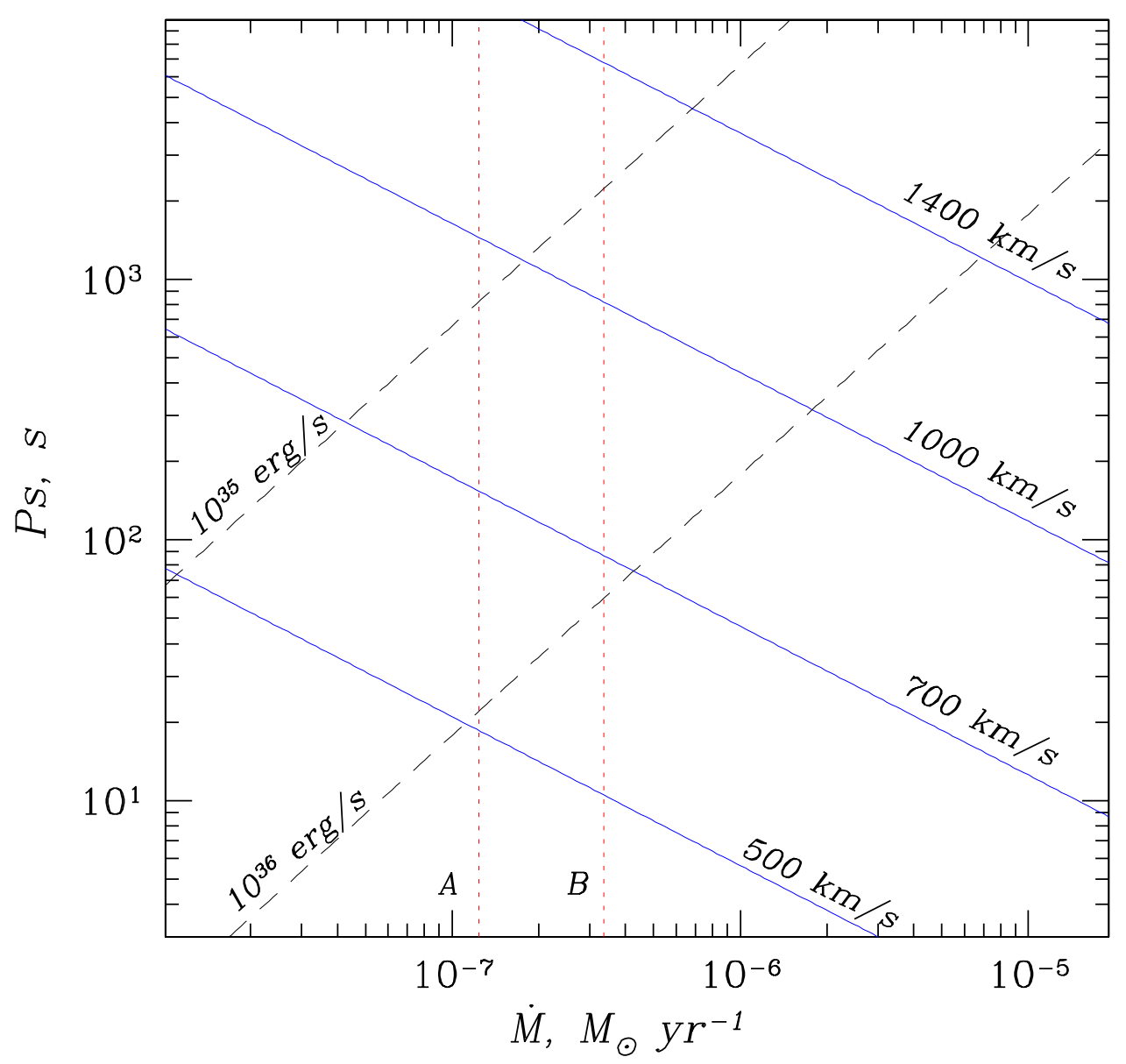

Fig. 10. Range of possible spin periods of the neutron star in IGR J17544-2619 as a function of the outflow rate of the stellar wind from the optical star. The solid (blue) lines indicate the equilibrium periods under different assumptions about the wind velocity. The dashed lines indicate the maximum achievable X-ray luminosities (in the absence of matter accumulation). The vertical (red) dotted lines indicate two estimates (see the text) of the wind outflow rate for a single OB star with the same parameters as those for the optical star of this binary system.

In Fig.10 the solid lines indicate $P_{s}^{*}$ as a function of the wind outflow rate $\dot{M}_{W}$ at the measured parameters for IGR J17544-2619 and under different assumptions about the wind velocity. It allows the range of values for the as yet unknown period of the pulsar in this binary system to be estimated. The vertical dotted lines in this figure indicate the expected outflow rate of the stellar wind from a single OB star with the same parameters as those for the optical component of the system. Lines A and B correspond to the formulas from Vink et al. (2001) and van Buren (1985), respectively. The dashed lines indicate the maximum possible X-ray luminosities of the source during outbursts (in the model by Grebenev and Sunyaev (2007), in the absence of matter accumulation in the period between outbursts). At a known observed luminosity these lines bound the wind velocity. The figure shows that our estimates of the parameters of the system and the optical star, on the whole, agree well with the above SFXT model. The coincidence with the model becomes even better during the periastron passage by the neutron star in this system. Figure11 (similar to Fig.(10) shows the maximum possible X-ray luminosty of the source and its spin period for this epoch under different assumptions about the wind outflow rate and velocity. The luminosity of about $10^{37} \mathrm{erg} \mathrm{s}^{-1}$ can be easily reached here by the neutron star with a period $\lesssim 10 \mathrm{~s}$. At the same time, the peak luminosity of $3 \times 10^{38} \mathrm{erg} \mathrm{s}^{-1}$ recorded by Romano et al. (2015) cannot be reached in the discussed range of wind outflow rates that implies the ejection of more dense matter by the star at that moment. We also see that the wind velocity cannot be very high (cannot exceed greatly the parabolic velocity $\left.\sqrt{2 G M / R} \sim 1000 \mathrm{~km} \mathrm{~s}^{-1}\right)$. How- 


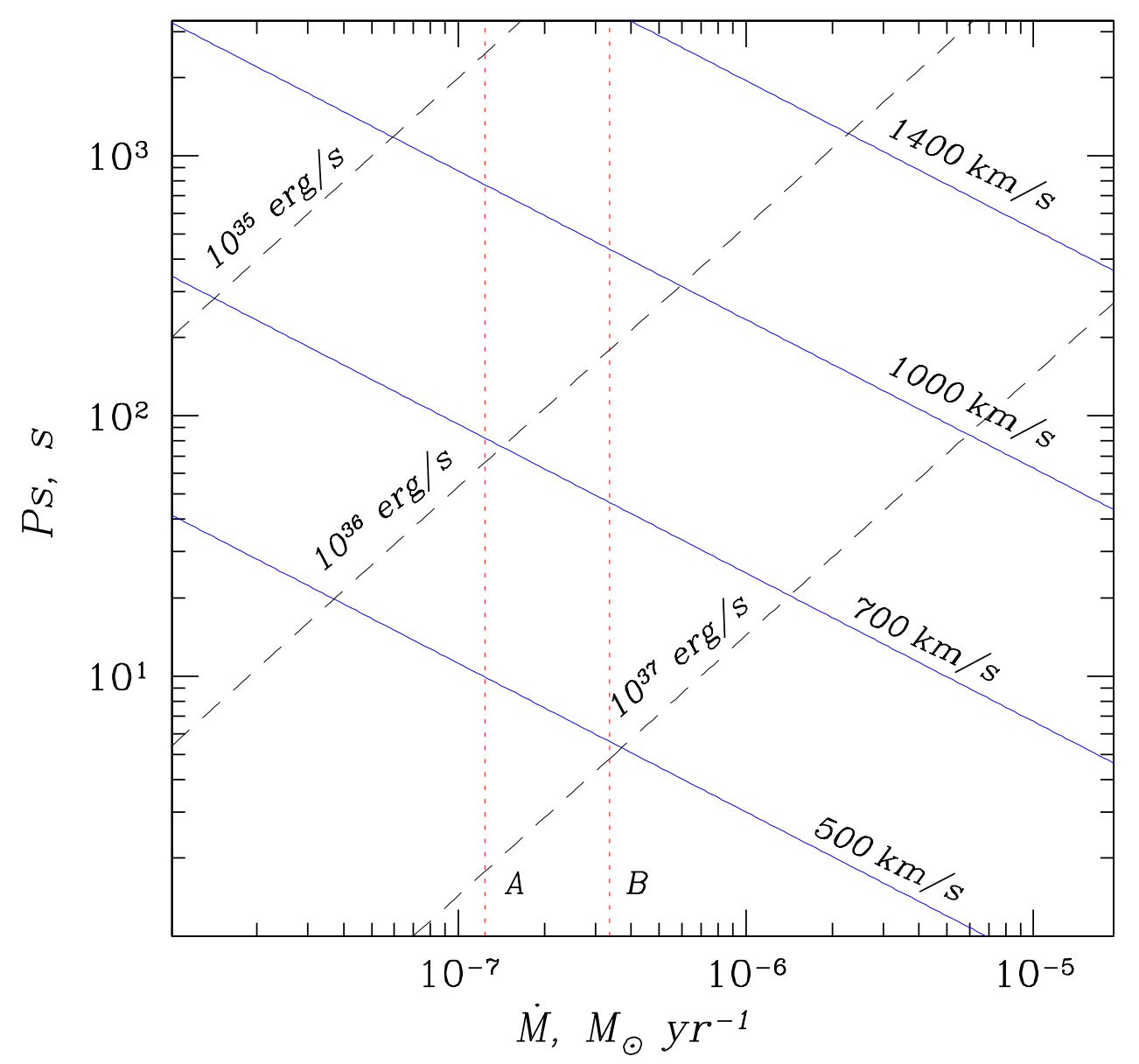

Fig. 11. The same as Fig.10 but during the periastron passage by the neutron star. The rate of accretion onto the neutron star and its luminosity are $\sim 3$ times higher than the average ones in the orbit (shown in Fig.10) at the same wind outflow rate. The spin periods shown by the blue lines are the equilibrium periods for the given accretion rate and may be slightly smaller than the actual period of the neutran star in IGR J17544-2619.

ever, the wind outflow rates estimated from the observations of single OB stars (Vink et al. 2001; van Buren 1985) can be underestimated for the star that is a component of an X-ray binary. According to the observations by Bozzo et al. (2016), a weak (with $\left.L_{X} \sim 10^{32}-10^{34} \mathrm{erg} \mathrm{s}^{-1}\right) \mathrm{X}$-ray emission is recorded from the neutron star of this system even in the period between outbursts; it can heat up the surface of the optical star and induce a stronger outflow of its wind.

\section{ACKNOWLEDGMENTS}

This work was financially supported by the Russian Foundation for Basic Research (project no. 1632-50071), the Government of Tatarstan (scientific project no. 15-42-02573), the "Transitional and Explosive Processes in Astrophysics" Subprogram of the Basic Research Program P-7 of the Presidium of the Russian Academy of Sciences, and though subsidy
3.6714.2017/8.9 granted to the Kazan Federal University to perform a State task in the area of scientific activity. We thank TUBITAK, KFU, AST, and IKI for partial support in using RTT-150 (the RussianTurkish 1.5-m telescope in Antalya).

\section{REFERENCES}

1. E. Anders and N. Grevesse, Geochim. Cosmochim. Acta 53, 197 (1989).

2. V. Bhalerao, P. Romano, J. Tomsick, L. Natalucci, D.M. Smith, E. Bellm, S.E. Boggs, D. Chakrabarty, et al., Mon. Not. Roy. Astron. Soc. 447, 2274 (2015).

3. E. Bozzo, M. Falanga, and L. Stella, Astrophys. J. 683, 1031 (2008).

4. E. Bozzo, V. Bhalerao, P. Pradhan, J. Tomsick, P. Romano, C. Ferrigno, S. Chaty, L. Oskinova, et al., Astron. Astrophys. 596, 16, (2016). 
5. D. van Buren, Astrophys. J. 294, 567 (1985).

6. F. Castelli and R.L. Kurucz, IAU Symp. 210, A20 (2004).

7. D.J. Clark, A.B. Hill, A.J. Bird, V.A. McBride, S. Scaringi, and A.J. Dean, Mon. Not. Roy. Astron. Soc. 399, 113 (2009).

8. T.J. Deeming, Astrophys. Space Sci. 36, 1, 137 (1975).

9. S.P. Drave, A.J. Bird, L.J.Townsend, A.B. Hill, V.A. McBride, V. Sguera, A. Bazzano, and D.J. Clark, Astron. Astrophys. 539, A21 (2012).

10. S.P. Drave, A.J. Bird, L. Sidoli, V. Sguera, A. Bazzano, A.B. Hill, and M.E. Goossenset, Mon. Not. Roy. Astron. Soc. 439, 2175 (2014).

11. G. A. Galazutdinov, Preprint SAO RAN 92, 1 (1992).

12. G. A. Galazutdinov, http://www.gazinur.com (2007).

13. A. Gimenez-Garcia, T. Shenar, J.M. Torrejon, L. Oskinova, S. Martinez-Nunez, W.-R. Hamann, J.J. Rodes-Roca, A. Gonzalez-Galan, J. Alonso-Santiago, C. Gonzalez-Fernandez, et al., Astron. Astrophys. $\mathbf{5 9 1}, 25$ (2016).

14. S.A. Grebenev, in Proceedings of the Conference "The Extreme Sky: Sampling the Universe above $10 \mathrm{keV",}$ Otranto, Italy, October 13-17, Proc. Sci. 096, 60 ( 2009)

15. S.A. Grebenev and R.A. Sunyaev, Astron. Lett. 33, 149 (2007).

16. S.A. Grebenev, A.A. Lutovinov, and R.A. Sunyaev, Astron. Tel. 192, 1 (2003).

17. S.A. Grebenev, J. Rodriguez, N.J. Westergaard, R.A. Sunyaev, and T. Oosterbroek, Astron. Tel. 252, 1 (2004).

18. H.R. Griem, Astrophys. J. 132, 883 (1960).

19. A.F. Illarionov and R.A. Sunyaev, Astron. Astrophys. 39, 185 (1975)

20. R.L. Kurucz, SAO CD-ROMs (Cambridge, MA, USA, 1994).

21. R.L. Kurucz and I. Furenlid, SAO Spec. Rep. 387, 1 (1979).

22. J. Lafler and T.D. Kinman, Astrophys. J. Suppl. Ser. 11, 216 (1965).

23. F. Lebrun, J.P. Leray, P. Lavocat, J. Cretolle, M. Arques, C. Blondel, C. Bonnin, A. Bouere, C. Cara, T. Chaleil, et al., Astron. Astrophys. 411, 141 (2003).

24. N.R. Lomb, Astrophys. Space Sci. 39, L447 (1976)

25. F. Martins, A. Palacios, Astron. Astrophys. 560, A16 (2012).

26. V.S. Menzhevitski, N.N. Shimanskaya, V.V. Shimansky, and D.O. Kudryavtsev, Astrophys. Bull., 69, 169 (2014).

27. I. Negueruela, D.M. Smith, P. Reig, S. Chaty, and J.M. Torrejon, ESA-SP 604, 165 (2006).

28. E.A. Nikolaeva, I.F. Bikmaev, S.S. Mel'nikov, et al. Byull. KrAO 109, 27 (2013).

29. E.A. Nikolaeva, I.F. Bikmaev, V.V. Shimansky, et al.,
ASP Conf. Ser. 510, 431 (2017).

30. B. Paczynski, Ann. Rev. Astron. Astrophys. 9, 183 (1971).

31. L.J. Pellizza, S. Chaty, and I. Negueruela, Astron. Astrophys. 455, 653 (2006).

32. F. Rahoui, S. Chaty, P.-O. Lagage, and E. Pantin, Astron. Astrophys. 484, 801 (2008).

33. P. Romano, C.B. Markwardt, M.M. Chester, V. Mangano, P.A. Evans, J.A. Kennea, H.A. Krimm, S.D. Barthelmy, et al., Astron. Tel. 5388, 1 (2013).

34. P. Romano, L. Ducci, V. Mangano, P. Esposito, E. Bozzo, and S. Vercellone, Astron. Astrophys. 568, A55 (2014).

35. P. Romano, E. Bozzo, V. Mangano, P. Esposito, G. Israel, A. Tiengo, S. Campana, L. Ducci, C. Ferrigno, and J. A. Kennea, Astron. Astrophys. 576, L4 (2015).

36. N. A. Sakhibullin, Tr. Kazan. Astron. Observ. 48, 9 (1983).

37. J.D. Scargle, Astrophys. J. 263, 835 (1982).

38. V. Sguera, A. Bazzano, A.J. Bird, A.J. Dean, P.Ubertini, E.J. Barlow, L. Bassani, D.J. Clark, et al., Astrophys. J. 646, 452 (2006).

39. N. Shakura, K. Postnov, L. Sidoli, and A. Paizis, Mon. Not. Roy. Astron. Soc. 442, 2325 (2014).

40. V.V. Shimansky, N.V. Borisov, and N.N. Shimanskaya, Astron. Rep. 47, 763 (2003).

41. V.V. Shimansky, E.A. Karitskaya, G.A. Bochkarev, V.M. Galazutdinov, V.M. Lyuty, and N.N. Shimanskaya, Astron. Rep. 56, 741 (2012).

42. L. Sidoli, P. Romano, S. Mereghetti, A. Paizis, S.Vercellone, V. Mangano, and D. Goötz, Astron. Astrophys. 476, 1307 (2007).

43. D.M. Smith, Astron. Tel. 6227, 1 (2014).

44. D.M. Smith, W.A. Heindl, J.H. Swank, T.E. Harrison, and I. Negueruela, Astron. Tel. 182, 1 (2003).

45. D.M. Smith, W.A. Heindl, C.B. Markwardt, J. H. Swank, I. Negueruela, T. E. Harrison, and L. Huss, Astrophys. J. 638, 974 (2006).

46. V.F. Suleymanov, Astron. Astrophys. Trans. 2, 197 (1992).

47. R. Sunyaev, A. Lutovinov, S. Molkov, and S. Deluit, Astron. Tel. 181, 1 (2003a).

48. R.A. Sunyaev, S.A. Grebenev, A.A. Lutovinov, J. Rodriguez, S. Mereghetti, D. Götz, and T. Courvoisier, Astron. Tel. 190, 1 (2003b).

49. P. Ubertini, F. Lebrun, G. Di Cocco, A. Bazzano, A.J. Bird, K. Broenstad, A. Goldwurm, G. La Rosa, C. Labanti, P. Laurent, et al., Astron. Astrophys. 411, L131 (2003).

50. A. Unsold, Physik der Sternatmospheren (Springer, Berlin-Gottingen-Heidelberg, 1955).

51. C.R. Vidal, J. Cooper, and E.W. Smith, Astrophys. J. Suppl. Ser. 25, 37 (1973).

52. J.S. Vink, A. de Koter, and H. Lamers, Astron. Astrophys. 369, 574 (2001). 
53. N.R. Walborn, E.L. Fitzpatrick, Publ. Astron. Soc. Pacific 102, 37 (1990).

54. C. Winkler, T. J.-L. Courvoisier, G. Di Cocco, N. Gehrels, A. Gimenez, S. Grebenev, W. Hermsen, J.M.
Mas-Hesse, F. Lebrun, N. Lund, et al., Astron. Astrophys. 411, 1, (2003).
55. N. Zacharias, C.T. Finch, T.M. Girard, et al., VizieR Online Data Catalog: I/322 (2012).

56. J.J.M. in't Zand, Astron. Astrophys. 441, 1 (2005).

57. J. in't Zand, J. Heise, P. Ubertini, A. Bazzano, and C. Markwardt, ESA-SP 552, 427 (2004).

Translated by V. Astakhov 